\title{
The impact of loneliness on healthcare use in older people: evidence from a nationally representative cohort
}

\author{
Annette Burns ${ }^{1,2}$ (I) $\cdot$ Gerard Leavey $^{2} \cdot$ Mark Ward $^{3} \cdot$ Roger O'Sullivan ${ }^{1,2}$
}

Received: 13 December 2019 / Accepted: 7 June 2020 / Published online: 22 June 2020

(C) The Author(s) 2020

\begin{abstract}
Aim Concerns around loneliness leading to increased healthcare use persist in spite of a mixed evidence base and lack of adjustment for key potential confounders. We investigated the associations among loneliness, health and healthcare use in older adults including stratification to investigate whether these associations differ by gender.

Subject and methods Secondary analysis of a nationally representative sample of 8175 community-dwelling adults aged 50 years and over from The Irish Longitudinal Study on Ageing (TILDA). Primary outcomes were self-reported general practitioner (GP) and emergency department (ED) visits in the past 12 months. Negative binomial and logistic regression analysis was used to investigate associations between loneliness and healthcare use, later adjusting for potential mediators (health and health behaviours).

Results Loneliness was consistently positively associated with number of GP visits according to both cross-sectional and longitudinal analyses, with incidence rate ratios (IRRs) ranging from 1.08 to 1.33 in the sample overall. Associations with ED visits were less consistent. After adjusting for health and health behaviours, male loneliness does not appear to influence ED or GP visits. However, women who reported loneliness had an elevated risk of an ED visit at wave 1 (W1; odds ratio (OR) 1.08 [1.01-1.16]), as well as increased GP visits at both waves (IRRs ranging from 1.05 [1.02-1.07] to 1.16 [1.07-1.26]).

Conclusion Older women experiencing loneliness visit their GP more often irrespective of health, health behaviours or social isolation. While effect sizes were small, there are implications for health service resources at a population level. Importantly, however, this may also be a useful opportunity to redirect towards appropriate services and tailored resources.
\end{abstract}

Keywords Loneliness $\cdot$ Healthcare use $\cdot$ Emergency department $\cdot$ General practitioner $\cdot$ Older adults $\cdot$ Primary care physician

\section{Introduction}

Although loneliness is experienced by all age groups, research has tended to focus on older adults for whom the prevalence of intense loneliness (often or always lonely) is consistently estimated at 8-9\% (Victor et al. 2002). Other studies employing more sensitive measures or lower thresholds of loneliness,

Annette Burns

annette.burns@publichealth.ie

1 The Institute of Public Health in Ireland, 700 South Circular Road, Dublin 8, Ireland

2 Bamford Centre for Mental Health and Wellbeing, Ulster University, Coleraine, Ireland

3 The Irish Longitudinal Study on Ageing, Trinity College Dublin, Dublin, Ireland which include those reporting that they are sometimes lonely, have found higher reported prevalence rates ranging between $25 \%$ and $57 \%$ in adults aged 60 years and over based on data from the USA and China (Gerst-Emerson and Jayawardhana 2015; Zhang et al. 2018). Loneliness is associated with poorer health, including depression, anxiety, heart disease (Valtorta et al. 2016), health behaviours such as physical activity (Hawkley et al. 2009) and premature mortality (HoltLunstad et al. 2015), with evidence especially strong for depression (Cacioppo et al. 2010). However, twin concerns about usage of health services by an ageing population and increasing loneliness raise concerns that loneliness may increase healthcare use (HCU).

Evidence to date in relation to whether loneliness is associated with HCU is mixed. Previous studies have found positive associations with general practitioner (GP) visits/consultations/contacts (Almind et al. 1991; Cheng 1992; Ellaway et al. 1999; Gerst-Emerson and Jayawardhana 2015; Newall 
et al. 2015; Taube et al. 2015; Theeke 2010; Wang et al. 2019; Zhang et al. 2018) and emergency department (ED) visits and hospitalisations (Geller et al. 1999; Molloy et al. 2010; Taube et al. 2015), as well as with outpatient care visits in general in one study (Taube et al. 2015). However, these associations are often weak or only explain a small amount of the variance, e.g. 11\% (Almind et al. 1991; Taube et al. 2015), and, at times, no association with HCU [planned hospitalisations (GerstEmerson and Jayawardhana 2015; Molloy et al. 2010); GP home visits (Ellaway et al. 1999); admission to hospital (Newall et al. 2015)] or, in the case of one study, a significant negative association was found, with significantly lower odds of physician visits among older adults who were lonely in Singapore (Lim and Chan 2017). In spite of the established associations between loneliness and health, however, few studies have examined if associations between loneliness and HCU are independent of health and none have ever adjusted for health behaviours. While several studies have reported associations independent of health for physician visits (Cheng 1992; Ellaway et al. 1999; Gerst-Emerson and Jayawardhana 2015; Wang et al. 2019; Zhang et al. 2018) and hospitalisations (Molloy et al. 2010; Zhang et al. 2018), most are cross-sectional, used a single item to assess loneliness and conducted only limited mediation analysis. Furthermore, no study had stratified these associations by gender, despite the known effects of gender on HCU (RedondoSendino et al. 2006). There is a considerable literature on helpseeking, particularly relating to service contacts and mental health (Curran et al. 2020), much of which is focussed on factors associated with stigma and avoidance (Greenley and Mullen 1990; Leavey et al. 2016; Mojtabai et al. 2002). Additionally, masculine attitudes and beliefs are considered to play a determining role in avoiding or rejecting help (Biddle et al. 2007). Various behavioural theories have been posited to assist explanation for help-seeking (or not). For example, the health belief model suggests that help-seeking is influenced by an individual's assessment of the potential risks posed by the health problem and its severity, balanced against benefits and ability to cope (Henshaw and FreedmanDoan 2009). Given that beliefs related to explanations of distress and coping are often culturally embedded, rational explanations for help-seeking may not be easily attainable (Kleinman 2004).

We hypothesised that loneliness would be associated with increased HCU in older adults, but that this would likely be explained by health and health behaviours. Given the literature to date on help-seeking, we also hypothesised that loneliness might be less associated with increased HCU in men. This paper tests these hypotheses by assessing whether loneliness predicts $\mathrm{HCU}$ in a nationally representative sample of community-dwelling adults aged 50 years and over; testing whether these associations, if present, are mediated by health and health behaviours; and, finally, investigating whether these associations and their potential mediators differ when we stratify by gender given the known effects of gender on HCU and help-seeking.

\section{Methods}

\section{Secondary analysis of TILDA data between W1 and W3}

The sample comprised participants from The Irish Longitudinal Study on Ageing (https://tilda.tcd.ie/), which provides a stratified, clustered, nationally representative sample of community-dwelling adults aged 50 years and over living in Ireland. Private residential dwellings were assigned to clusters stratified by geography and socio-economic group to produce a population-representative sample. Across households where it was possible to make contact to confirm eligibility, a response rate of $62 \%$ was achieved, with all residents aged 50 years and over in each household invited to participate (Barrett et al. 2011). The current analysis involves waves 1-3. Data collection involved an extensive face-to-face, computer-assisted home interview, a self-completion questionnaire for data deemed more sensitive and a health assessment at wave 1 (W1) and wave 3 (W3) (see Table 1 for an overview of relevant data collected in TILDA and modes of collection, as well as sample sizes).

\section{Exposure variables}

1. Loneliness was assessed in the self-completion questionnaire using the five-item UCLA scale, which is a revised version of the 20-item University of California-Los Angeles (UCLA) Loneliness Scale (Russell et al. 1978). Each item is measured on a three-point Likert scale (0-2) reflecting frequency of occurrence: 'hardly ever or never'; 'some of the time'; 'often'. Possible scores range from 0 to 10 , with higher scores indicating greater loneliness. This score was used as an indicator of loneliness in the current study, as were two additional generated variables based on items $1-3$ and item 5 . to allow comparability with previous papers, as well as to observe associations for chronic loneliness as below.

2. To allow comparability, a loneliness threshold variable was created in line with previous papers establishing the impact of loneliness on HCU (Gerst-Emerson and Jayawardhana 2015; Lim and Chan 2017), whereby any participant responding 'often' or 'some of the time' to any of the first three items was defined as lonely. This threshold variable was also employed to observe chronic loneliness, i.e. participants meeting this criteria at all three waves. 
Table 1 Relevant data collected by The Irish Longitudinal Study on Ageing (TILDA) for adults aged 50 years and over

\begin{tabular}{|c|c|c|c|c|c|}
\hline Wave & Data collected & Age (years) & $\begin{array}{l}\text { Computer-assisted } \\
\text { personal interview }\end{array}$ & Returned a self-completion questionnaire & Completed health assessment \\
\hline 1 & 2009-2011 & $50+$ & 8173 & $6913(85 \%)$ & $5893(72 \%)$ \\
\hline 2 & $2012-2013$ & $52+$ & 6993 & $5888(84 \%)$ & N/A \\
\hline 3 & 2014-2015 & $54+$ & 6248 & $5216(83 \%)$ & $5043(81 \%)$ \\
\hline \multicolumn{3}{|c|}{ Data included in the current analyses: } & $\begin{array}{l}\text { - Demographics (W1) } \\
\text { - GP visits (W1, W3) } \\
\text { - ED visits (W1, W3) } \\
\text { - Chronic conditions (W1, W3) } \\
\text { - Falls (W1, W3) } \\
\text { - Pain (W1, W3) } \\
\text { - CES-D (W1, W3) } \\
\text { - IPAQ (W1, W3) } \\
\text { - Smoking status (W1, W3) }\end{array}$ & $\begin{array}{l}\text { - UCLA (W1, W2, W3) } \\
\text { - CAGE (W1, W3) } \\
\text { - HADS-A (W1, W3) }\end{array}$ & $\begin{array}{l}\text { - BMI (W1, W3) } \\
\text { - Waist circumference (W1, W3) }\end{array}$ \\
\hline
\end{tabular}

3. Finally, responses of 'some of the time' or 'often' to UCLA item 5, which asks directly about how often respondents 'feel lonely', were also modelled at wave 1 and at wave 3 as an indicator of chronic loneliness.

\section{Outcomes}

Primary outcomes were: GP and ED visits as self-reported during computer-assisted face-to-face personal interview at wave 1 and wave 3 . Binary categorical outcome variables were also generated to capture report of at least one ED visit in the previous 12 months at $\mathrm{W} 1$ and at $\mathrm{W} 3$.

\section{Potential mediators}

Variables indicating health status and, therefore, adjusted for in the mediation analysis were: presence of a self-reported doctor-diagnosed chronic condition (79.7\%); reporting 'troubled often with pain' (35\%) or a fall in the last year (19.4\%); body mass index (BMI) (mean 28.6, standard deviation [SD] 4.94); waist circumference (mean $95.4 \mathrm{~cm}, \mathrm{SD} 13.9$ ); and scales assessing anxiety (Hospital Anxiety and Depression Scale-Anxiety subscale, HADS-A) ( $7.2 \%$ probable) (Zigmond and Snaith 1983) and depressive (Center for Epidemiologic Studies Depression Scale, CES-D) (9.5\% severe) symptoms, with the CES-D short form eight-item version used at wave 3 with a validated cut-off of 9 (Briggs et al. 2018). Health behaviours included were smoking status (current (18\%)/former (38\%)/never); alcohol problem as per the CAGE questionnaire (12\%) (score of 2 or more) (Mayfield et al. 1974); and physical activity, with the sample split into three groups using the short form eight-item version of the International Physical Activity Questionnaire (IPAQ) (Craig et al. 2003).
Doctor-diagnosed chronic conditions included in TILDA waves 1 and 3 were: cancer; chronic lung disease; cardiovascular disease (angina; high cholesterol; hypertension; diabetes; myocardial infarction or coronary thrombosis; congestive heart failure; a stroke; or transient ischemic attack); asthma; arthritis; osteoporosis; Parkinson's disease; 'any emotional, nervous or psychiatric problems'; alcohol or substance abuse; Alzheimer's disease; dementia; serious memory impairment; cataracts; glaucoma; age-related macular degeneration; stomach ulcers; cirrhosis/serious liver damage; and varicose ulcers. For the purposes of this analysis, the presence of at least one chronic condition was defined as answering 'yes' when asked if they were 'ever told by a doctor that' they had any of the above. Those who refused to answer or reported they did not know were treated as missing.

\section{Statistical analyses}

Key variables and demographic characteristics of the sample were compared according to threshold and direct item loneliness at wave 1 and chronic threshold and direct item loneliness at wave 3 using $t$-test and chi-square statistics as appropriate.

At wave 1, loneliness was modelled as: (1) UCLA score; (2) threshold variable based on UCLA items 1-3; and (3) UCLA item 5, which asks directly about feeling lonely. Longitudinally chronic loneliness was defined as: (1) meeting threshold based on UCLA items 1-3 at all three waves and (2) reporting 'often' or 'some of the time' to UCLA item 5 at all three waves.

Multivariate negative binomial and logistic regression models were used to investigate associations between loneliness and GP visits (count) and between loneliness and ED visits (count; any versus none) cross-sectionally at wave 1 and longitudinally at wave 3 based on chronic loneliness. All models were weighted and adjusted for age (continuous), 
sex, education and marital status and multilevel to account for non-independence at the household level. Negative binomial regression was employed for count data as an alternative to Poisson, as it is useful for count data with overdispersion (i.e. sample variance is higher than the sample mean).

Potential mediators (health and health behaviours) were added to the model at step 2 to check for mediation effects. For longitudinal models, variables indicating health and health behaviours at wave 3 were employed. Due to missing data, the final analytic samples for full mediation analysis ranged from 4256 to 4308 at wave 1 and from 3621 to 3682 at wave 3 .

Finally, all models were stratified by gender to observe differences in associations or mediation according to gender.

\section{Results}

Overall, the mean UCLA score for the five-item scale at wave 1 was 1.95 (SD 2.18, median 1) $(n=6689)$. Among the 6829 individuals for whom UCLA items 1-3 were available, $48 \%$ ( $n=3278)$ were defined as lonely, i.e. answered 'sometimes' or 'often' to at least one of these three items. In the case of UCLA item 5, meanwhile, which directly asks about loneliness, $35 \%(n=2410 / 6805)$ reported that they felt lonely 'some of the time' $(29 \%)$ or 'often' $(6 \%)$.

In relation to chronic loneliness, based on the threshold variable, $26.7 \%(n=1170 / 4380)$ were lonely at all three waves, while the prevalence was $17.0 \%(n=757 / 4463)$ based on UCLA item 5.

The mean number of self-reported GP visits in the last 12 months was 3.99 (SD 5.44, median 3) $(n=8162)$ and 3.98 (SD 5.24, median 3) $(n=6225)$ at wave 1 and wave 3 , respectively. The mean number of self-reported ED visits in the last 12 months was 0.26 (SD 1.18, median 0$)(n=8165)$ and 0.25 (SD 0.68 , median 0$)(n=6238)$. Overall, $15 \%$ reported at least one ED visit at wave 1, while $17.6 \%$ reported an ED visit at wave 3 .

Table 2 illustrates the main characteristics of the sample at wave 1 based on threshold loneliness. Meeting the 'lonely' threshold was significantly related to gender, age group, education, marital status, number of GP visits and reporting at least one ED visit but not the overall number of ED visits. It was also significantly related to all health and health behaviour variables except BMI and waist circumference. BMI and waist circumference were, however, associated with number of GP visits (and an ED visit/none in the case of waist circumference) and were, therefore, retained in mediation models. Loneliness as per UCLA item 5 was associated with all except BMI, waist circumference and mean age.

Chronic threshold loneliness was associated with GP visits, health behaviours and all socio-demographic and health variables except mean age, education, BMI and waist circumference.
Chronic loneliness based on UCLA item 5 was not associated with age (mean/group) or BMI.

Loneliness was significantly associated with both GP and ED visits at wave 1 across indicators of loneliness (Table 3 ). The exception was number of ED visits, which was associated with UCLA score but not threshold loneliness or direct item loneliness. Following the addition of health and health behaviours to these models, all associations between loneliness and ED visits became non-significant. However, loneliness remained significantly independently associated with GP visits, in the case of both UCLA score and the loneliness threshold variable.

The stratification of models and mediation analysis by gender revealed that, while loneliness was consistently associated with GP visits in both men and women with incidence rate ratios (IRRs) ranging from 1.07 to 1.33 , an association with number of ED visits was only present for men and only in relation to UCLA score, while significant associations with an ED visit compared to none were only consistently found for women. Following the addition of health and health behaviours to these stratified models, however, all associations between loneliness and HCU in men became non-significant. Loneliness remained a significant independent predictor of GP visits in women across indicators and of reporting an ED visit based on UCLA score only.

As shown in Table 4, chronic loneliness was consistently predictive of GP visits at wave 3, with chronic direct item loneliness predictive of ED visits also. Once health and health behaviours were added, however, only the association between threshold loneliness and GP visits remained (IRR $1.10,95 \%$ confidence interval [CI] (1.01-1.19)).

The stratification of longitudinal models by gender revealed that associations between chronic loneliness and HCU were, again, fully mediated in men by health and health behaviours and the only independent significant association present was in women in relation to GP visits and based on threshold loneliness variable.

\section{Discussion}

\section{Key findings}

The data indicate that loneliness was consistently positively associated with number of GP visits according to both crosssectional and longitudinal analyses, with IRRs ranging from 1.08 to 1.33 in the sample overall. Associations with ED visits were less consistent and, while reporting at least one ED visit at wave 1 was consistently associated with loneliness indicators, the number of ED visits was only associated with UCLA score in the cross-sectional analysis. At wave 3 , only chronic loneliness based on direct item predicted ED visits (count/one or none), while chronic threshold loneliness showed no association. 
Table 2 Demographics, health and healthcare use characteristics of The Irish Longitudinal Study on Ageing (TILDA) cohort by loneliness (threshold variable based on items $1-3)(n=6829)$

\begin{tabular}{|c|c|c|c|c|c|c|}
\hline & \multicolumn{2}{|c|}{ Lonely, $n=3278,48 \%$} & \multicolumn{2}{|c|}{ Not lonely, $n=3551,52 \%$} & \multirow[t]{2}{*}{$t /$ chi } & \multirow[t]{2}{*}{$p$-Value } \\
\hline & No. & $\%$ & No. & $\%$ & & \\
\hline \multicolumn{7}{|l|}{ Gender $(n=6829)$} \\
\hline Male & 1423 & $46 \%$ & 1687 & $54 \%$ & 11.5 & $0.001 *$ \\
\hline Female & 1855 & $50 \%$ & 1864 & $50 \%$ & & \\
\hline Age, years $($ mean $(\mathrm{SD}))(n=6820)$ & 63.8 & $(9.85)$ & 63.4 & $(9.33)$ & 3.01 & 0.08 \\
\hline \multicolumn{7}{|l|}{ Education $(n=6827)$} \\
\hline Primary/none & 964 & $51 \%$ & 934 & $49 \%$ & 8.84 & $0.012 *$ \\
\hline Secondary & 1326 & $47 \%$ & 1470 & $53 \%$ & & \\
\hline Third/higher & 987 & $46 \%$ & 1146 & $54 \%$ & & \\
\hline \multicolumn{7}{|l|}{ Marital status $(n=6829)$} \\
\hline Married & 1944 & $40 \%$ & 2907 & $60 \%$ & 422.7 & $<0.001 * *$ \\
\hline Never married & 416 & $66 \%$ & 212 & $34 \%$ & & \\
\hline Separated/divorced & 296 & $69 \%$ & 130 & $31 \%$ & & \\
\hline Widowed & 622 & $67 \%$ & 302 & $33 \%$ & & \\
\hline \multicolumn{7}{|l|}{ Healthcare use } \\
\hline GP visits past 12 months (mean (SD)) $(n=6822)$ & 4.49 & $(6.17)$ & 3.41 & $(4.70)$ & -8.11 & $<0.001 * *$ \\
\hline ED visits past 12 months (mean (SD)) $(n=6825)$ & 0.28 & $(1.19)$ & 0.023 & $(1.27)$ & -1.58 & 0.113 \\
\hline ED visit (at least one) $(n=1008 / 6825)$ & 533 & $53 \%$ & 475 & $47 \%$ & 11.4 & $0.001 *$ \\
\hline Doctor-diagnosed chronic condition $(n=5457 / 6816)$ & 2700 & $49.5 \%$ & 2757 & $50.5 \%$ & 22.9 & $<0.001 * *$ \\
\hline Pain ('often troubled with') $(n=2404 / 6825)$ & 1362 & $57 \%$ & 1042 & $43 \%$ & 11.8 & $<0.001 * *$ \\
\hline Fall(s) (in past year) $(n=1342 / 6827)$ & 724 & $54 \%$ & 618 & $46 \%$ & 23.6 & $<0.001 * *$ \\
\hline Waist circumference (mean (SD)) $(n=5294)$ & 95.3 & $(14.0)$ & 95.3 & $(13.6)$ & 0.02 & 0.894 \\
\hline BMI (mean (SD)) $(n=5302)$ & 28.6 & $(5.22)$ & 28.5 & $(4.69)$ & -0.3585 & 0.720 \\
\hline \multicolumn{7}{|l|}{ CES-D depressive symptoms $(n=6725)$} \\
\hline Severe $(16+)$ & 488 & $82 \%$ & 110 & $18 \%$ & 541.0 & $<0.001 * *$ \\
\hline Moderate (8-15) & 764 & $65 \%$ & 418 & $35 \%$ & & \\
\hline None/mild (7 or less) & 1959 & $40 \%$ & 1959 & $60 \%$ & & \\
\hline \multicolumn{7}{|l|}{ HADS-A $(n=6584)$} \\
\hline Probable $(11+)$ & 472 & $80 \%$ & 116 & $20 \%$ & 542.3 & $<0.001 * *$ \\
\hline Possible (8-10) & 686 & $68 \%$ & 328 & $32 \%$ & & \\
\hline Normal (7 or less) & 1974 & $40 \%$ & 3008 & $60 \%$ & & \\
\hline Alcohol problem (CAGE) $(n=810 / 6692)$ & 461 & $57 \%$ & 349 & $43 \%$ & 31.0 & $<0.001 * *$ \\
\hline \multicolumn{7}{|l|}{ Smoking $(n=6829)$} \\
\hline Never & 1418 & $47 \%$ & 1623 & $53 \%$ & 20.3 & $<0.001 * *$ \\
\hline Past & 1232 & $47 \%$ & 1393 & $53 \%$ & & \\
\hline Current & 628 & $54 \%$ & 535 & $46 \%$ & & \\
\hline \multicolumn{7}{|l|}{ Exercise (IPAQ) $(n=6762)$} \\
\hline Low & 1094 & $52 \%$ & 1014 & $48 \%$ & 25.3 & $<0.001^{* *}$ \\
\hline Moderate & 1140 & $48 \%$ & 1224 & $52 \%$ & & \\
\hline High & 1015 & $44 \%$ & 1275 & $56 \%$ & & \\
\hline
\end{tabular}

$*<.05 ; * *<.001$

Following the addition of health and health behaviours, loneliness had no impact on ED visits in the sample overall, based on both cross-sectional and longitudinal models. For GP visits, however, loneliness remained significantly independently associated in both cross-sectional and longitudinal analyses, based on total score and threshold loneliness at wave 1 and based on chronic threshold loneliness at wave 3, with IRRs ranging from 1.03 to 1.11 . Loneliness, therefore, appeared to be an independent predictor of GP visits across the sample. 
Table 3 Associations between loneliness and healthcare use at wave 1 including mediation analysis

\begin{tabular}{|c|c|c|c|c|c|c|c|c|c|}
\hline & \multirow[t]{2}{*}{ GP visits (count) } & \multirow[t]{2}{*}{ No. } & \multirow[t]{2}{*}{ IRR } & \multirow[t]{2}{*}{$95 \% \mathrm{CI}$} & \multirow[t]{2}{*}{$p$-Value } & \multicolumn{4}{|c|}{ Adjusted for health status (objective) } \\
\hline & & & & & & No. & Adjusted IRR & $95 \% \mathrm{CI}$ & $p$-Value \\
\hline 1 & \multirow[t]{3}{*}{ UCLA score } & 6671 & 1.08 & $(1.06-1.09)$ & $<0.001 * *$ & 4256 & 1.03 & $(1.01-1.05)$ & $0.004 *$ \\
\hline Men & & 3065 & 1.07 & $(1.04-1.10)$ & $<0.001 * *$ & 1946 & 1.01 & $(0.98-1.04)$ & 0.668 \\
\hline Women & & 3606 & 1.07 & $(1.06-1.09)$ & $<0.001 * *$ & 2310 & 1.05 & $(1.02-1.07)$ & $<0.001 * *$ \\
\hline 2 & \multirow[t]{3}{*}{ UCLA threshold } & 6811 & 1.27 & $(1.18-1.36)$ & $<0.001 * *$ & 4304 & 1.11 & $(1.03-1.20)$ & $0.007 *$ \\
\hline Men & & 3105 & 1.17 & $(1.05-1.31)$ & $0.005^{*}$ & 1960 & 1.04 & $(0.91-1.18)$ & 0.584 \\
\hline Women & & 3706 & 1.33 & $(1.22-1.45)$ & $<0.001 * *$ & 2344 & 1.16 & $(1.07-1.26)$ & $<0.001 * *$ \\
\hline 3 & \multirow[t]{3}{*}{ Direct item } & 6789 & 1.30 & $(1.21-1.40)$ & $<0.001 * *$ & 4299 & 1.06 & $(0.97-1.15)$ & 0.204 \\
\hline Men & & 3101 & 1.25 & $(1.11-1.41)$ & $<0.001 * *$ & 1960 & 0.95 & $(0.84-1.09)$ & 0.482 \\
\hline \multirow[t]{2}{*}{ Women } & & 3688 & 1.33 & $(1.22-1.45)$ & $<0.001 * *$ & 2339 & 1.14 & $(1.03-1.26)$ & $0.011 *$ \\
\hline & ED visits (count) & No. & IRR & $95 \% \mathrm{CI}$ & $p$-Value & & Adjusted IRR & $95 \% \mathrm{CI}$ & $p$-Value \\
\hline 1 & \multirow[t]{3}{*}{ UCLA score } & 6675 & 1.06 & $(1.02-1.11)$ & $0.003 *$ & 4260 & 0.99 & $(0.93-1.06)$ & 0.874 \\
\hline Men & & 3065 & 1.07 & $(1.02-1.13)$ & $0.010^{*}$ & 1947 & 1.01 & $(0.94-1.08)$ & 0.856 \\
\hline Women & & 3610 & 1.05 & $(0.99-1.12)$ & 0.109 & 2313 & 1.01 & $(0.93-1.10)$ & 0.824 \\
\hline 2 & \multirow[t]{3}{*}{ UCLA threshold } & 6814 & 1.16 & $(0.92-1.47)$ & 0.202 & 4308 & 0.82 & $(0.63-1.07)$ & 0.137 \\
\hline Men & & 3105 & 1.05 & $(0.80-1.38)$ & 0.707 & 1961 & 0.89 & $(0.65-1.21)$ & 0.453 \\
\hline Women & & 3709 & 1.23 & $(0.86-1.76)$ & 0.256 & 2347 & 0.82 & $(0.58-1.16)$ & 0.260 \\
\hline 3 & \multirow[t]{3}{*}{ Direct item } & 6792 & 1.24 & $(0.98-1.56)$ & 0.073 & 4303 & 0.90 & $(0.66-1.22)$ & 0.487 \\
\hline Men & & 3101 & 1.16 & $(0.89-1.51)$ & 0.273 & 1961 & 1.00 & $(0.74-1.35)$ & 0.981 \\
\hline \multirow[t]{2}{*}{ Women } & & 3691 & 1.29 & $(0.92-1.81)$ & 0.141 & 2342 & 0.93 & $(0.64-1.34)$ & 0.692 \\
\hline & ED visit (1 or more) & No. & ORR & $95 \% \mathrm{CI}$ & $p$-Value & & Adjusted ORR & $95 \% \mathrm{CI}$ & $p$-Value \\
\hline 1 & \multirow[t]{3}{*}{ UCLA score } & 6675 & 1.07 & $(1.03-1.10)$ & $<0.001 * *$ & 4260 & 1.04 & $(0.98-1.09)$ & 0.169 \\
\hline Men & & 3065 & 1.06 & $(1.01-1.11)$ & $0.015^{*}$ & 1947 & 1.00 & $(0.92-1.08)$ & 0.923 \\
\hline Women & & 3610 & 1.07 & $(1.02-1.12)$ & $0.004 *$ & 2313 & 1.08 & $(1.01-1.16)$ & $0.028 *$ \\
\hline 2 & \multirow[t]{3}{*}{ UCLA threshold } & 6814 & 1.18 & $(1.01-1.37)$ & $0.035^{*}$ & 4308 & 1.03 & $(0.84-1.26)$ & 0.761 \\
\hline Men & & 3105 & 1.10 & $(0.89-1.37)$ & 0.379 & 1961 & 0.96 & $(0.71-1.30)$ & 0.799 \\
\hline Women & & 3709 & 1.25 & $(1.01-1.54)$ & $0.036^{*}$ & 2347 & 1.14 & $(0.85-1.51)$ & 0.383 \\
\hline 3 & \multirow[t]{3}{*}{ Direct item } & 6792 & 1.25 & $(1.08-1.46)$ & $0.003 *$ & 4303 & 1.13 & $(0.90-1.41)$ & 0.306 \\
\hline Men & & 3101 & 1.21 & $(0.97-1.51)$ & 0.098 & 1961 & 1.07 & $(0.77-1.49)$ & 0.690 \\
\hline Women & & 3691 & 1.29 & $(1.05-1.59)$ & $0.017 *$ & 2342 & 1.18 & $(0.87-1.59)$ & 0.296 \\
\hline
\end{tabular}

All models weighted and adjusted for age (continuous), sex, education and marital status

$\mathrm{CI}$, confidence interval; IRR, incidence rate ratio

$*<.05 ; * *<.001$

The stratification of models by gender revealed consistent associations for both men and women in relation to GP visits. In relation to ED visits, differences emerged in the crosssectional analyses, with no association present for women in relation to number of ED visits and no association for men in relation to reporting one or more ED visits based on threshold or direct item loneliness variables. Following the addition of health and health behaviours to stratified models, however, all associations between loneliness and HCU in men became non-significant, both cross-sectionally and longitudinally. In women, meanwhile, loneliness remained independently significantly associated with GP visits at wave 1 (based on all indicators) and wave 3 (based on chronic threshold loneliness), with IRRs ranging from 1.05 to 1.16. An association with $\mathrm{ED}$ visits in terms of reporting one or more visit also remained for women based on UCLA score only. Importantly, these associations also remained following the addition of social connection score (Berkman and Syme 1979) to the model, suggesting that it was loneliness rather than social isolation that was responsible for these associations with $\mathrm{HCU}$ in women (data not shown).

\section{Strengths}

Our findings are based on a large, nationally representative sample of older adults and the inclusion of longitudinal 
Table 4 Associations between chronic loneliness and healthcare use at wave 3 including mediation analysis

\begin{tabular}{|c|c|c|c|c|c|c|c|c|c|}
\hline & \multirow[t]{2}{*}{ GP visits (count) } & \multirow[t]{2}{*}{ No. } & \multirow[t]{2}{*}{ IRR } & \multirow[t]{2}{*}{$95 \% \mathrm{CI}$} & \multirow[t]{2}{*}{$p$-Value } & \multicolumn{4}{|c|}{ Adjusted for health status } \\
\hline & & & & & & No. & Adjusted IRR & $95 \% \mathrm{CI}$ & $p$-Value \\
\hline \multirow[t]{3}{*}{1} & Chronically lonely 1 & 4369 & 1.28 & $(1.18-1.38)$ & $<0.001 * *$ & 3621 & 1.10 & $(1.01-1.19)$ & $0.028 *$ \\
\hline & Men & 1954 & 1.28 & $(1.12-1.47)$ & $<0.001 * *$ & 1650 & 1.10 & $(0.97-1.24)$ & 0.140 \\
\hline & Women & 2415 & 1.25 & $(1.15-1.36)$ & $<0.001 * *$ & 1971 & 1.11 & $(1.01-1.23)$ & $0.030 *$ \\
\hline \multirow[t]{4}{*}{2} & Chronically lonely 2 & 4452 & 1.33 & $(1.21-1.47)$ & $<0.001 * *$ & 3680 & 1.03 & $(0.94-1.13)$ & 0.544 \\
\hline & Men & 1995 & 1.45 & $(120-1.76)$ & $<0.001 * *$ & 1680 & 1.09 & $(0.93-1.27)$ & 0.288 \\
\hline & Women & 2457 & 1.23 & $(1.11-1.36)$ & $<0.001 * *$ & 2000 & 1.01 & $(0.90-1.12)$ & 0.914 \\
\hline & ED visits (count) & No. & IRR & $95 \% \mathrm{CI}$ & $p$-Value & No. & Adjusted IRR & $95 \% \mathrm{CI}$ & $p$-Value \\
\hline \multirow[t]{3}{*}{1} & Chronically lonely 1 & 4375 & 1.11 & $(0.90-1.36)$ & 0.333 & 3624 & 0.88 & $(0.70-1.11)$ & 0.277 \\
\hline & Men & 1956 & 1.08 & $(0.78-1.50)$ & 0.643 & 1651 & 0.89 & $(0.62-1.29)$ & 0.553 \\
\hline & Women & 2419 & 1.15 & $(0.89-1.48)$ & 0.278 & 1973 & 0.88 & $(0.68-1.15)$ & 0.362 \\
\hline \multirow[t]{4}{*}{2} & Chronically lonely 2 & 4457 & 1.47 & $(1.16-1.85)$ & $0.001 *$ & 3682 & 1.25 & $(0.95-1.65)$ & 0.117 \\
\hline & Men & 1997 & 1.56 & $(1.06-2.28)$ & $0.024 *$ & 1681 & 1.35 & $(0.83-2.20)$ & 0.226 \\
\hline & Women & 2460 & 1.42 & $(1.07-1.87)$ & $0.014 *$ & 2001 & 1.22 & $(0.90-1.64)$ & 0.194 \\
\hline & ED visit (1 or more) & No. & IRR & $95 \% \mathrm{CI}$ & $p$-Value & No. & Adjusted ORR & $95 \% \mathrm{CI}$ & $p$-Value \\
\hline \multirow[t]{3}{*}{1} & Chronically lonely 1 & 4375 & 1.11 & $(0.90-1.37)$ & 0.311 & 3624 & 0.87 & $(0.68-1.11)$ & 0.271 \\
\hline & Men & 1956 & 1.06 & $(0.77-1.46)$ & 0.730 & 1651 & 0.78 & $(0.52-1.17)$ & 0.225 \\
\hline & Women & 2419 & 1.16 & $(0.89-1.52)$ & 0.271 & 1973 & 0.94 & $(0.68-1.29)$ & 0.685 \\
\hline \multirow[t]{3}{*}{2} & Chronically lonely 2 & 4457 & 1.47 & $(1.17-1.85)$ & $0.001 *$ & 3682 & 1.29 & $(0.97-1.73)$ & 0.085 \\
\hline & Men & 1997 & 1.51 & $(1.04-2.20)$ & $0.031 *$ & 1681 & 1.24 & $(0.76-2.00)$ & 0.389 \\
\hline & Women & 2460 & 1.47 & (1.09-1.97) & $0.010^{*}$ & 2001 & 1.36 & $(0.94-1.96)$ & 0.101 \\
\hline
\end{tabular}

All models weighted and adjusted for age (continuous), sex, education and marital status

Chronically lonely 1 : respondents who met threshold categorical variable based on UCLA items $1-3$ at all three waves $(n=1593)$

Chronically lonely 2 : respondents who reported feeling 'sometimes' or 'often' lonely at all three waves as per direct item [UCLA item 5] ( $n=763$ )

analyses based on three waves of data. TILDA, with its robust methodology, provides a detailed and rich populationweighted dataset and the necessary power to adjust for many confounders (Barrett et al. 2011).

The inclusion of the five-item UCLA in TILDA also meant that we were able to look at loneliness based on data beyond the widely used three-item UCLA, using the direct item measure provided by item 5 , and a total score, including this, as well as item 4, a reverse-scored positively worded item. In the context of a current lack of consensus on a validated UCLA cut-off (Fried et al. 2020), this approach allowed us to look across these indicators for consistent patterns relating to $\mathrm{HCU}$ while also providing data comparable to other recent longitudinal analyses on loneliness and HCU (Gerst-Emerson and Jayawardhana 2015; Lim and Chan 2017).

In adjusting for health status, a full range of variables capturing a comprehensive picture of objective physical and mental health were included, as were health behaviours (smoking status, physical activity, alcohol use) that have not, to our knowledge, been looked at before in adjusting for associations between loneliness and HCU, in spite of known associations with both (Hawkley et al. 2009; Lauder et al. 2006). This was also the first paper, to our knowledge, to stratify associations between loneliness and HCU by gender, in spite of established effects of gender on HCU (Redondo-Sendino et al. 2006).

\section{Limitations}

While, as above, it was helpful to observe associations based on a definition of loneliness used in recent studies on $\mathrm{HCU}$ (Gerst-Emerson and Jayawardhana 2015; Lim and Chan 2017), the threshold variable could be argued to provide an overly broad definition of loneliness, which is perhaps overly sensitive, and this could also be argued in relation to the inclusion of those responding 'some of the time' to the direct item. However, the fact that, unlike feeling 'often' lonely, feeling 'sometimes' lonely does appear to be increasing over time in older adults (Hawkley et al. 2019; Victor et al. 2002) supports this broader approach. If it is this less intense loneliness that appears to be on the rise in society, then investigation of its potential consequences is important. Given the stigma associated with loneliness (Lau and Gruen 1992), it is also possible that it may be under-reported, although its placement in the self-completion questionnaire should have somewhat 
tempered this. Arguably, some models were overfitted due to the inclusion of the CAGE questionnaire under health behaviours (which assesses the presence of drinking problems) and lifetime diagnosis of alcohol/substance abuse under chronic conditions. However, given that these were slightly different indicators, the low prevalence in the sample $(1.6 \%$ reported diagnosed alcohol/substance abuse at W1), the lack of any multicollinearity problems detected by Stata and the fact that they were both included as potential mediators at all times (rather than predictors or outcomes), it was decided to retain both to ensure a full mediation analysis. The self-report of $\mathrm{HCU}$ also means that these data may be subject to recall bias, even though the period in question was limited to the previous 12 months only. Additionally, while the Berkman-Syme Social Network Index (SNI; Berkman and Syme 1979) did not, in this case, mediate the association between loneliness and HCU in women, further exploration using more comprehensive measures of social isolation in future studies would be useful. Finally, it is also possible that other health indicators, not included in TILDA, may explain the associations between loneliness and $\mathrm{HCU}$ found in women.

\section{Loneliness and healthcare use}

Unlike their male counterparts, women experiencing loneliness consistently reported more GP visits independent of their health and health behaviours.

This is in line with a number of studies that found associations between loneliness and physician visits independent of health (Ellaway et al. 1999; Gerst-Emerson and Jayawardhana 2015; Wang et al. 2019; Zhang et al. 2018), including one study that was limited to older women (aged 65-85 years) (Cheng 1992).

In contrast to the current study, Molloy et al. also found an independent association with emergency hospitalisations in a community sample of Irish adults. However, this was based on cross-sectional data, a single-item measure of loneliness and a limited mediation analysis that relied on a single-item assessment of chronic illness (Molloy et al. 2010). In China, Zhang et al. also found an independent association with annual hospitalisations but, again, these were cross-sectional data, a single-item loneliness measure and a reliance on simple binary outcomes with no count data available (Zhang et al. 2018). The current study omitted planned hospitalisations and looked only at ED visits based on the evidence in the literature of a lack of association with planned hospitalisations (GerstEmerson and Jayawardhana 2015; Molloy et al. 2010) compared to ED visits and admissions (Geller et al. 1999; Molloy et al. 2010; Taube et al. 2015). As the first study on loneliness and HCU to stratify by gender, this paper indicates that the role of gender is important and may have a key role in making sense of the mixed evidence base to date.
While not previously examined in relation to loneliness and $\mathrm{HCU}$, gender differences have been demonstrated with regard to numerous other potentially related health issues. For instance, there are known to be significant differences between men and women in relation to symptom reporting, with women reporting more intense, more numerous and more frequent symptoms compared to men (Barsky et al. 2001). Despite this generally increased symptom reporting, however, research has also demonstrated that women may be less likely to receive diagnoses, as has been shown in the case of stroke, for instance ( $\mathrm{Yu}$ et al. 2019). In the current study, we controlled for doctor-diagnosed conditions as part of a mediation analysis to see if health might explain associations between loneliness and increased HCU, but if women were less likely to receive these diagnoses, this could have impacted our results. Notably, health-related quality of life and number of diseases have also been shown to be important in relation to increased HCU in women (Redondo-Sendino et al. 2006) and the current study, which adjusted based on presence of disease only, may, therefore, have missed this cumulative effect.

Another possible explanation for the association between loneliness and HCU remaining independent in women in this paper is the role of employment status, given that this sample included adults aged 50 years and over and social contact through work is not captured by the SNI (which is based on marital status; contact with children, relatives and friends; church group membership; and membership in other voluntary organisations) (Berkman and Syme 1979). In Ireland, women aged 50 years and over are less likely to be working than men of the same age (Ward 2019) and this may, in part, be due to the marriage bar introduced by the Irish government in 1932, which required women to leave paid employment on marriage and remained in place for civil servants until 1973 (Mosca and Wright 2020). Previous analysis of the TILDA dataset has revealed that women affected by the Irish marriage bar have shorter working lives, lower individual income, higher household wealth at present, more children and more educated children, yet, are not significantly different to women unaffected in relation to current health status (Mosca and Wright 2020). In line with other studies on loneliness and $\mathrm{HCU}$, we note that the size of the independent effects found was not large (Almind et al. 1991; Taube et al. 2015) and while there are implications for health services resources at a population level, this may also represent a valuable opportunity to intervene. Future research should seek to explore whether the relationships found in the current study hold within other cultural and health system settings. Further exploration of the role of potential mechanisms such as functional limitations and depression, in relation to directions of effects, is also needed. Evidence in relation to what might work as an effective loneliness screener for GPs and also in relation to concordance between GP and patients' own perception of patient loneliness would also be helpful. Current evidence 
suggests that GPs' ability to identify patients who are lonely is limited (Due et al. 2018). Finally, further qualitative work is needed to better understand the experience of loneliness as well as its associations with HCU from both patient and healthcare professional perspectives.

Our study is the first to date to explore the role of gender in associations between loneliness and HCU and its mediation by health. It is also the first to include health behaviours and the first to explore these relationships across three waves of population-level data. We observed a consistent association between loneliness and GP visits in women independent of health and health behaviours. These findings lead us to conclude that GPs may be well placed to refer or redirect older women experiencing loneliness towards appropriate services and tailored resources.

Acknowledgements The authors would like to acknowledge the contribution of the participants in the study, members of The Irish Longitudinal Study on Ageing (TILDA) research team and administrators.

Funding TILDA was supported by the Irish government, the Atlantic Philanthropies and Irish Life PLC. This secondary analysis paper forms part of a joint programme of research which is funded by the Institute of Public Health in Ireland (IPH) and Ulster University.

\section{Compliance with ethical standards}

As this study involved only secondary analyses of the TILDA dataset, separate ethical approval for this study was not necessary. However, ethical approval for the TILDA project was sought and gained from the Faculty of Health Sciences Research Ethics Committee, Trinity College Dublin. Written informed consent to participate in TILDA was obtained from all participants as part of the initial screening that preceded the study interview.

Conflict of interest The authors declare that they have no conflict of interest.

Open Access This article is licensed under a Creative Commons Attribution 4.0 International License, which permits use, sharing, adaptation, distribution and reproduction in any medium or format, as long as you give appropriate credit to the original author(s) and the source, provide a link to the Creative Commons licence, and indicate if changes were made. The images or other third party material in this article are included in the article's Creative Commons licence, unless indicated otherwise in a credit line to the material. If material is not included in the article's Creative Commons licence and your intended use is not permitted by statutory regulation or exceeds the permitted use, you will need to obtain permission directly from the copyright holder. To view a copy of this licence, visit http://creativecommons.org/licenses/by/4.0/.

\section{References}

Almind G, Holstein BE, Holst E, Due P (1991) Old persons' contact with general practitioners in relation to health: a Danish population study. Scand J Prim Health Care 9:252-258. https://doi.org/10.3109/ 02813439109018528
Barrett A, Burke H, Cronin H et al (2011) Fifty plus in Ireland 2011: first results from the Irish longitudinal study on ageing (TILDA). The Irish Longitudinal Study on Ageing, Trinity College Dublin, Dublin

Barsky AJ, Peekna HM, Borus JF (2001) Somatic symptom reporting in women and men. J Gen Intern Med 16:266-275. https://doi.org/10. 1046/j.1525-1497.2001.016004266.x

Berkman LF, Syme SL (1979) Social networks, host resistance, and mortality: a nine-year follow-up study of Alameda County residents. Am J Epidemiol 109:186-204

Biddle L, Donovan J, Sharp D, Gunnell D (2007) Explaining non-helpseeking amongst young adults with mental distress: a dynamic interpretive model of illness behaviour. Sociol Health Illn 29:9831002. https://doi.org/10.1111/j.1467-9566.2007.01030.x

Briggs R, Carey D, O'Halloran AM, Kenny RA, Kennelly SP (2018) Validation of the 8-item Centre for Epidemiological Studies Depression Scale in a cohort of community-dwelling older people: data from The Irish Longitudinal Study on Ageing (TILDA). Eur Geriatr Med 9:121-126

Cacioppo JT, Hawkley LC, Thisted RA (2010) Perceived social isolation makes me sad: 5-year cross-lagged analyses of loneliness and depressive symptomatology in the Chicago Health, Aging, and Social Relations Study. Psychol Aging 25:453-463

Cheng ST (1992) Loneliness-distress and physician utilization in wellelderly females. J Community Psychol 20:43-56

Craig CL, Marshall AL, Sjöström M et al (2003) International physical activity questionnaire: 12-country reliability and validity. Med Sci Sports Exerc 35:1381-1395

Curran E, Rosato M, Ferry F, Leavey G (2020) Prevalence and factors associated with anxiety and depression in older adults: gender differences in psychosocial indicators. J Affect Disord 267:114-122. https://doi.org/10.1016/j.jad.2020.02.018

Due TD, Sandholdt H, Siersma VD, Waldorff FB (2018) How well do general practitioners know their elderly patients' social relations and feelings of loneliness? BMC Fam Pract 19:34. https://doi.org/10. 1186/s12875-018-0721-x

Ellaway A, Wood S, Macintyre S (1999) Someone to talk to? The role of loneliness as a factor in the frequency of GP consultations. Br J Gen Pract 49:363-367

Fried L, Prohaska T, Burholt V et al (2020) A unified approach to loneliness. Lancet 395:114

Geller J, Janson P, McGovern E, Valdini A (1999) Loneliness as a predictor of hospital emergency department use. J Fam Pract 48:801804

Gerst-Emerson K, Jayawardhana J (2015) Loneliness as a public health issue: the impact of loneliness on health care utilization among older adults. Am J Public Health 105:1013-1019. https://doi.org/10.2105/ AJPH.2014.302427

Greenley JR, Mullen JA (1990) Help-seeking and the use of mental health services. Res Community Ment Health 6:325-350

Hawkley LC, Thisted RA, Cacioppo JT (2009) Loneliness predicts reduced physical activity: cross-sectional \& longitudinal analyses. Health Psychol 28:354-363. https://doi.org/10.1037/a0014400

Hawkley LC, Wroblewski K, Kaiser T, Luhmann M, Schumm LP (2019) Are U.S. older adults getting lonelier? Age, period, and cohort differences. Psychol Aging 34:1144-1157. https://doi.org/10.1037/ pag0000365

Henshaw EJ, Freedman-Doan CR (2009) Conceptualizing mental health care utilization using the health belief model. Clin Psychol Sci Pract 16:420-439. https://doi.org/10.1111/j.1468-2850.2009.01181.x

Holt-Lunstad J, Smith TB, Baker M, Harris T, Stephenson D (2015) Loneliness and social isolation as risk factors for mortality: a meta-analytic review. Perspect Psychol Sci 10:227-237

Kleinman A (2004) Culture and depression. N Engl J Med 351:951-953. https://doi.org/10.1056/NEJMp048078

Lau S, Gruen GE (1992) The social stigma of loneliness: effect of target person's and perceiver's sex. Pers Soc Psychol Bull 18:182-189 
Lauder W, Mummery K, Jones M, Caperchione C (2006) A comparison of health behaviours in lonely and non-lonely populations. Psychol Health Med 11:233-245. https://doi.org/10.1080/13548500500266607

Leavey G, Rosato M, Galway K, Hughes L, Mallon S, Rondon J (2016) Patterns and predictors of help-seeking contacts with health services and general practitioner detection of suicidality prior to suicide: a cohort analysis of suicides occurring over a two-year period. BMC Psychiatry 16:120

Lim KK, Chan A (2017) Association of loneliness and healthcare utilization among older adults in Singapore. Geriatr Gerontol Int 17: 1789-1798. https://doi.org/10.1111/ggi.12962

Mayfield D, McLeod G, Hall P (1974) The CAGE questionnaire: validation of a new alcoholism screening instrument. Am J Psychiatry 131:1121-1123. https://doi.org/10.1176/ajp.131.10.1121

Mojtabai R, Olfson M, Mechanic D (2002) Perceived need and helpseeking in adults with mood, anxiety, or substance use disorders. Arch Gen Psychiatry 59:77-84

Molloy GJ, McGee HM, O’Neill D, Conroy RM (2010) Loneliness and emergency and planned hospitalizations in a community sample of older adults. J Am Geriatr Soc 58:1538-1541. https://doi.org/10. 1111/j.1532-5415.2010.02960.x

Mosca I, Wright RE (2020) The long-term consequences of the Irish marriage Bar. Econ Soc Rev 51:1-34

Newall N, McArthur J, Menec VH (2015) A longitudinal examination of social participation, loneliness, and use of physician and hospital services. J Aging Health 27:500-518. https://doi.org/10.1177/ 0898264314552420

Redondo-Sendino Á, Guallar-Castillón P, Banegas JR, RodríguezArtalejo F (2006) Gender differences in the utilization of healthcare services among the older adult population of Spain. BMC Public Health 6:155

Russell D, Peplau LA, Ferguson ML (1978) "Developing a measure of loneliness." Journal of personality assessment 42(3):290-294

Taube E, Kristensson J, Sandberg M, Midlöv P, Jakobsson U (2015) Loneliness and health care consumption among older people. Scand J Caring Sci 29:435-443. https://doi.org/10.1111/scs.12147

Theeke LA (2010) Sociodemographic and health-related risks for loneliness and outcome differences by loneliness status in a sample of
U.S. older adults. Res Gerontol Nurs 3:113-125. https://doi.org/ 10.3928/19404921-20091103-99

Valtorta NK, Kanaan M, Gilbody S, Ronzi S, Hanratty B (2016) Loneliness and social isolation as risk factors for coronary heart disease and stroke: systematic review and meta-analysis of longitudinal observational studies. Heart 102:1009-1016. https://doi.org/ 10.1136/heartjnl-2015-308790

Victor CR, Scambler SJ, Shah S et al (2002) Has loneliness amongst older people increased? An investigation into variations between cohorts. Ageing Soc 22:585-597

Wang H, Zhao E, Fleming J, Dening T, Khaw KT, Brayne C (2019) Is loneliness associated with increased health and social care utilisation in the oldest old? Findings from a population-based longitudinal study. BMJ Open 9:e024645. https://doi.org/10.1136/bmjopen2018-024645

Ward M (2019) Irish adults transition to retirement - wellbeing, social participation and health-related behaviours. Findings from The Irish Longitudinal Study on Ageing (TILDA). The Irish Longitudinal Study on Ageing, Trinity College Dublin, Dublin. Available online at: https://tilda.tcd.ie/publications/reports/pdf/Report_Retirement. pdf. Accessed 9 Oct 2019

Yu AYX, Penn AM, Lesperance ML et al (2019) Sex differences in presentation and outcome after an acute transient or minor neurologic event. JAMA Neurol 76:962-968. https://doi.org/10.1001/ jamaneurol.2019.1305

Zhang J, Xu L, Li J et al (2018) Loneliness and health service utilization among the rural elderly in Shandong, China: a cross-sectional study. Int J Environ Res Public Health 15:1468. https://doi.org/10.3390/ ijerph15071468

Zigmond AS, Snaith RP (1983) The hospital anxiety and depression scale. Acta Psychiatr Scand 67:361-370. https://doi.org/10.1111/j. 1600-0447.1983.tb09716.x

Publisher's note Springer Nature remains neutral with regard to jurisdictional claims in published maps and institutional affiliations. 